\title{
The Place Code of Saccade Metrics in the Lateral Bank of the Intraparietal Sulcus
}

\author{
Helen E. Savaki, ${ }^{1,2}$ Georgia G. Gregoriou, ${ }^{1,2}$ Sophia Bakola, ${ }^{1,2}$ Vassilis Raos, ${ }^{1,2}$ and Adonis K. Moschovakis ${ }^{1,2}$ \\ ${ }^{1}$ Institute of Applied and Computational Mathematics, Foundation for Research and Technology-Hellas, GR-71110 Iraklion, Crete, Greece, and \\ ${ }^{2}$ Department of Basic Sciences, Faculty of Medicine, School of Health Sciences, University of Crete, GR-71003 Iraklion, Crete, Greece
}

The lateral intraparietal area (LIP) of monkeys is known to participate in the guidance of rapid eye movements (saccades), but the means it uses to specify movement variables are poorly understood. To determine whether area LIP devotes neural space to encode saccade metrics spatially, we used the quantitative $\left[{ }^{14} \mathrm{C}\right]$ deoxyglucose method to obtain images of the distribution of metabolic activity in the intraparietal sulcus (IPs) of rhesus monkeys trained to repeatedly execute saccades of the same amplitude and direction for the duration of the experiment. Different monkeys were trained to perform saccades of different sizes and in different directions. A clear topography of saccade metrics was found in the cytoarchitectonically identified area LIP ventral (LIPv) contralateral to the direction of the eye movements. We demonstrate that the representation of the vertical meridian runs parallel to the fundus of the IPs and that it is not orthogonal to the representation of the horizontal meridian. Instead, the latter runs through the middle of LIPv parallel to its border with area LIP dorsal (LIPd). The upper part of oculomotor space is represented rostrally and dorsally relative to the horizontal meridian toward the LIPv-LIPd border, whereas the lower part of oculomotor space is represented caudally and ventrally toward the caudal edge of the IPs. Saccade amplitude is also represented in an orderly manner.

\section{Introduction}

One of the central goals of the neurosciences is to understand how the brain specifies movement variables. Realization of this goal is more likely to succeed first in the case of saccades because of their lawful time course and the relative simplicity of the mechanical apparatus that executes them. Their metrics are now known to be computed by a complex circuit made of several classes of neurons distributed through several brain regions. Close to system output, premotoneurons of the reticular formation use a time (and frequency) code to specify saccade amplitude and direction (Moschovakis et al., 1996). Such neurons discharge for all movements within $90^{\circ}$ of their on direction (left, right, up, or down), and it is the intensity and duration of their discharge that specifies movement size. In contrast, higher order structures such as the superior colliculus (SC) use a labeled line code to specify movement metrics. The deeper layers of the SC contain cells that discharge maximally for contraversive saccades of particular amplitude and direction (collectively defining the movement field of the neuron). It is only those SC neurons whose movement field contains the movement in question that are activated for its execution. The retinotopic coordinates of movement fields are further reflected in the territories of SC space

Received May 14, 2009; revised Aug. 25, 2009; accepted Aug. 26, 2009.

This work was supported by European Union Grants BI04-CT98-0546 and FP6 IST-027574, and PENED Grant $01 E D 111$ from the Greek General Secretariat of Research and Technology. We thank Maria Kefaloyianni for help with autoradiographic imaging.

Correspondence should be addressed to Adonis K. Moschovakis, Department of Basic Sciences, Faculty of Medicine, School of Health Sciences, University of Crete, P.0. Box 2208, GR-71003 Iraklion, Crete, Greece. E-mail: moschov@med.uoc.gr.

DOI:10.1523/JNEUROSCI.2268-09.2010

Copyright $\odot 2010$ the authors $\quad 0270-6474 / 10 / 301118-10 \$ 15.00 / 0$ occupied by the cells they belong to. Cells that prefer small saccades are located in the rostral SC, and cells preferring large saccades are located more caudally. Also, cells that discharge before upward saccades are located medially and cells discharging before downward saccades are located laterally (Wurtz and Goldberg, 1972). Accordingly, the SC can be said to use a place code to represent a movement variable (the vector of desired saccades).

The use of neural space to represent movement variables becomes less evident for structures upstream of the SC. For example, the frontal eye field (FEF) encodes saccade size in terms of the location of the active population within a small ( $\sim 6$-mm-long) region of the anterior bank of the arcuate sulcus (As); small saccades are represented laterally and ventrally toward its inferior limb, whereas large saccades are represented medially and dorsally toward its superior limb (Sommer and Wurtz, 2000). However, no topographic map of direction has been found in this portion of the FEF; nor is there any information about the spatial representation of movement variables within the much bigger area in the posterior bank of the As that also houses the FEF (Moschovakis et al., 2004).

The lateral intraparietal (LIP) area is a second case in point. It contains a representation of both the central and the peripheral visual field, but only its coarse topography has been described to date (Blatt et al., 1990; Ben Hamed et al., 2001). Neighboring neurons in it are said to display response fields with neighboring centers, but occasional abrupt discontinuities lead to uncertainty about its topographic organization (Schluppeck et al., 2005). To examine whether populations of LIP neurons encode movement variables spatially, in terms of the space they occupy, we sought to visualize their aggregate activity in rhesus monkeys performing saccades of a specific amplitude and direction with the 


\section{a Horizontal Saccades}

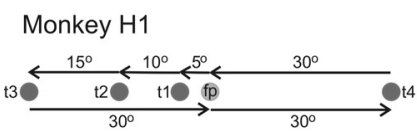

Monkey $\mathrm{H} 2$

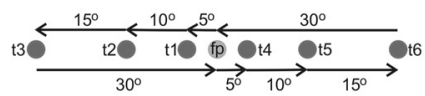

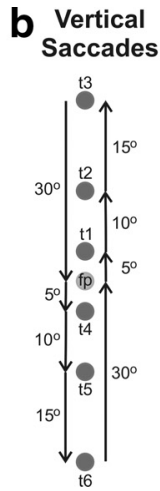
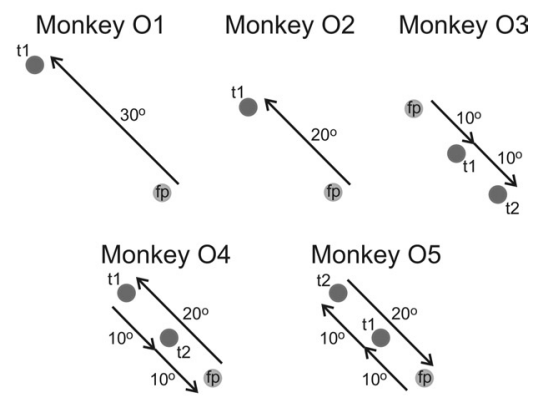

Figure 1. Behavioral tasks. $\boldsymbol{a}$, Monkey $\mathrm{H} 1$ performed a sequence of visually guided horizontal saccades $5^{\circ}$ (from the fixation point, fp to $\mathrm{t} 1$ ), $10^{\circ}$ (from $\mathrm{t} 1 \mathrm{to} \mathrm{t} 2$ ), and $15^{\circ}$ (from t2 to $\mathrm{t} 3$ ) to the left, then two saccades $30^{\circ}$ to the right (from $\mathrm{t} 3$ to $\mathrm{fp}$ and from fp to $\mathrm{t} 4$ ), and finally a saccade $30^{\circ}$ to the left (from t4 to fp). Monkey $\mathrm{H} 2$ performed a sequence of horizontal saccades $5^{\circ}$ (from fp to t 1 ), $10^{\circ}$ (from t 1 to $\mathrm{t} 2$ ), and $15^{\circ}$ (from t2 to t3) to the left, then one saccade $30^{\circ}$ to the right (from $\mathrm{t} 3 \mathrm{to} \mathrm{fp}$ ), then a sequence of saccades $5^{\circ}$ (from fp to $\mathrm{t} 4$ ), $10^{\circ}$ (from t 4 to t5), and $15^{\circ}$ (from t 5 to t6) to the right, and finally a saccade $30^{\circ}$ to the left (from t 6 to $\mathrm{fp}$ ). $\boldsymbol{b}$, A monkey performed a sequence of vertical upward saccades $5^{\circ}$ (from $\mathrm{fp}$ to $\mathrm{t} 1$ ), $10^{\circ}$ (from t1 to t2), and $15^{\circ}$ (from t2 to t3) in size, then one downward saccade $30^{\circ}$ in size (from t $3 \mathrm{to} f \mathrm{f}$ ), then a sequence of downward saccades $5^{\circ}$ (from fp to t4), $10^{\circ}$ (from t 4 to t5), and $15^{\circ}$ (from t5 to t6) in size, and finally an upward saccade $30^{\circ}$ in size (from t6 to fp). c, Monkeys 01 and 02 performed single oblique up-left ( $135^{\circ}$ in direction) saccades, 30 and $20^{\circ}$ in amplitude, respectively (from fp to t1). Monkey 03 executed a sequence of two down-right ( $10^{\circ}$ in amplitude and $315^{\circ}$ in direction) saccades (from fp to $\mathrm{t} 1$ and from $\mathrm{t} 1 \mathrm{to} \mathrm{t} 2$ ). Monkey 04 executed an up-left saccade, $20^{\circ}$ in amplitude and $135^{\circ}$ in direction (from fp to t 1 ) followed by a sequence of two down-right saccades, $10^{\circ}$ in amplitude and $315^{\circ}$ in direction (from t 1 to $\mathrm{t} 2$ and from $\mathrm{t} 2$ to fp). Monkey 05 executed two up-left visually guided saccades, $10^{\circ}$ in amplitude and $135^{\circ}$ in direction (from fp to $\mathrm{t} 1$ and from $\mathrm{t} 1 \mathrm{to} \mathrm{t}$ ) followed by one down-right saccade, $20^{\circ}$ in amplitude and $315^{\circ}$ in direction (from t2 to fp). Targets are shown as gray circles and saccades as black arrows.

\section{Table 1. Saccades executed by each monkey}

\begin{tabular}{|c|c|c|c|}
\hline & Saccade amplitude $\left(^{\circ}\right)$ & Direction & No. of saccades \\
\hline \multicolumn{4}{|c|}{ Horizontal saccades } \\
\hline \multirow[t]{5}{*}{ Monkey H1 } & 5 & 180 & 127 \\
\hline & 10 & 180 & 111 \\
\hline & 15 & 180 & 112 \\
\hline & 30 & 0 & 191 \\
\hline & 30 & 180 & 100 \\
\hline \multirow[t]{8}{*}{ Monkey H2 } & 5 & 180 & 72 \\
\hline & 10 & 180 & 53 \\
\hline & 15 & 180 & 61 \\
\hline & 30 & 0 & 50 \\
\hline & 5 & 0 & 90 \\
\hline & 10 & 0 & 75 \\
\hline & 15 & 0 & 67 \\
\hline & 30 & 180 & 62 \\
\hline \multirow[t]{8}{*}{ Vertical saccades } & 5 & 90 & 74 \\
\hline & 10 & 90 & 74 \\
\hline & 15 & 90 & 65 \\
\hline & 30 & 270 & 62 \\
\hline & 5 & 270 & 105 \\
\hline & 10 & 270 & 81 \\
\hline & 15 & 270 & 63 \\
\hline & 30 & 90 & 63 \\
\hline \multicolumn{4}{|l|}{ Oblique saccades } \\
\hline Monkey 01 & 30 & 135 & 217 \\
\hline Monkey 02 & 20 & 135 & 119 \\
\hline Monkey 03 & 10 & 315 & 212 \\
\hline \multirow[t]{2}{*}{ Monkey 04} & 20 & 135 & 130 \\
\hline & 10 & 315 & 331 \\
\hline \multirow[t]{2}{*}{ Monkey 05} & 10 & 135 & 323 \\
\hline & 20 & 315 & 156 \\
\hline
\end{tabular}

$\left[{ }^{14} \mathrm{C}\right]$ deoxyglucose $\left(\left[{ }^{14} \mathrm{C}\right] \mathrm{DG}\right)$ quantitative autoradiographic method (Sokoloff et al., 1977). The present report describes our findings in the lateral bank of the intraparietal sulcus (IPs).

\section{Materials and Methods}

Subjects. Twelve head-fixed adult female monkeys (Macaca mulatta) weighing between 3 and $5 \mathrm{~kg}$ were used in experiments complying with European Council Directive 86/609/EEC and in accordance with a protocol approved by the Animal Use Committee of the Foundation for Research and Technology-Hellas. Surgical procedures for implanting a head-holding metal bolt and a scleral search coil for monitoring the instantaneous position of the eyes were performed in sterile conditions and under general anesthesia as described previously (Moschovakis et al., 2001). Antibiotics and analgesics were administered systemically before and after surgery, and the monkeys were allowed to recover for $\sim 4$ weeks. Training lasted for $1 \mathrm{~h}$ per day during 3-6 months until the monkeys perfected their performance ( $\sim 95 \%$ success rate). Successful completion of trials was rewarded with water delivered through a tube attached close to their mouth. Monkeys had their arms restrained on a primate chair (CRIST Instrument) for the duration of the $\left[{ }^{14} \mathrm{C}\right] \mathrm{DG}$ experiment $(45 \mathrm{~min})$. All experimental tasks were performed in darkness. Eye position was sampled at a rate of $500 \mathrm{~Hz}$ using the Spike2 software (Cambridge Electronics Design). Only movements that obeyed the main sequence curve of the monkey were considered to represent saccades (Van Gisbergen et al., 1981).

Tasks. The behavioral apparatus was a video monitor placed $23 \mathrm{~cm}$ in front of the monkeys. Saccade targets were red circles, $1.5^{\circ}$ in diameter. Monkeys executing saccades to visual targets were required to hold eye position within a circular window $\left(2.5^{\circ}\right.$ in diameter) centered on the target. Each trial was initiated with the appearance of a central fixation target. The animals had to fixate it until it disappeared and a peripheral target was turned on signaling that a saccade to it should be executed within $1 \mathrm{~s}$. The minimum latency to move the eyes after the onset of each target was set to $0.1 \mathrm{~s}$ to discourage anticipatory movements. Monkeys had to fixate the peripheral target for $0.3-0.8 \mathrm{~s}$ until it disappeared. Animals were free to move their eyes spontaneously during the intertrial interval, which lasted for $0.2-1 \mathrm{~s}$.

Figure 1 provides a pictorial summary of the saccade vectors that our monkeys were trained to execute, whereas Table 1 lists the number of saccades each animal executed to each target during the first $10 \mathrm{~min}$ of the $\left[{ }^{14} \mathrm{C}\right] \mathrm{DG}$ experiment. This time period is critical because $\sim 85 \%$ of the radiolabeled DG is taken up before it ends (Sokoloff et al., 1977). Briefly, two animals were trained to execute horizontal saccades ( $\mathrm{H} 1$ and $\mathrm{H} 2$ ), one made vertical saccades, three made oblique saccades of 10,20 , or $30^{\circ}$ amplitude, respectively $(\mathrm{O} 1, \mathrm{O} 2$, and $\mathrm{O} 3)$, two (O4 and $\mathrm{O} 5)$ made oblique saccades of mixed amplitude $\left(10\right.$ and $\left.20^{\circ}\right)$, two were trained to fixate a central fixation point (Cf), and two animals were free to move their eyes in the dark (Cd). Three of these monkeys (one Cd, one Cf, and monkey O2) were used in the previous study by Bakola et al. (2006). Since the LIP participates in the execution of contraversive saccades (Gnadt and Andersen, 1988; Blatt et al., 1990), the hemispheres we studied $(N=21)$ were grouped separately depending on the direction of contralateral saccades.

One of the two monkeys executing horizontal saccades (Fig. 1a, monkey H1) was required to perform a sequence of 5,10 , and $15^{\circ}$ movements to the left, followed by two consecutive $30^{\circ}$ movements to the right and a $30^{\circ}$ movement to the left. Within the critical first $10 \mathrm{~min}$ of the $\left[{ }^{14} \mathrm{C}\right] \mathrm{DG}$ 
Table 2. Effects of saccade direction in LIPv

\begin{tabular}{llllrr}
\hline LIPv subregion & Cd & Cf & Experimental & Difference \\
representing $(n)$ & LCGU \pm SD & LCGU \pm SD & LCGU \pm SD & \multicolumn{1}{l}{$\%$ Cd } & \multicolumn{1}{l}{ \%Cf } \\
\hline Horizontal meridian (52) & $48.4 \pm 3.8$ & $48.8 \pm 3.4$ & $53.2 \pm 4.0$ & $\mathbf{9 . 9}$ & $\mathbf{9 . 0}$ \\
Vertical meridian (44) & $50.6 \pm 2.2$ & $49.9 \pm 3.1$ & $58.1 \pm 4.1$ & $\mathbf{1 4 . 9}$ & $\mathbf{1 6 . 5}$ \\
Oblique up saccades (70) & $49.2 \pm 1.9$ & $53.5 \pm 2.5$ & $58.0 \pm 2.0$ & $\mathbf{1 7 . 9}$ & $\mathbf{8 . 4}$ \\
Oblique down saccades (39) & $50.2 \pm 1.8$ & $49.7 \pm 2.8$ & $59.9 \pm 4.4$ & $\mathbf{1 9 . 3}$ & $\mathbf{2 0 . 5}$ \\
\hline
\end{tabular}

LCGU values (mean \pm SD) in micromoles $\cdot 100 \mathrm{~g}^{-1} \cdot$ minute ${ }^{-1}$ in different parts of LIPv activated for horizontal, vertical, oblique up, and oblique down saccades, respectively. $n$, Number of sets of five adjacent horizontal sections used to evaluate mean LCGU values. Difference $\% \mathrm{Cd}$, Percentage difference relative to $\mathrm{Cd}$ monkeys, measured as follows: (experimental $-\mathrm{Cd}) /\left(\mathrm{C}^{*} 100\right.$. Difference $\% \mathrm{Cf}$, Percentage difference relative to $\mathrm{Cf}$ monkeys, measured as follows: (experimental $-(\mathrm{f}) / \mathrm{ff}^{*} 100$. Values in bold indicate statistically significant differences tested with Student's unpaired $t$ test $(p<0.001)$.

Table 3. Effects of fixation in LIPd

\begin{tabular}{lllllll}
\hline Group & $\begin{array}{l}\text { LIPd (fix) (46) } \\
\text { LCGU } \pm \text { SD }\end{array}$ & $\begin{array}{l}\text { LIPd (all) (92) } \\
\text { LCGU } \pm \text { SD }\end{array}$ & $\begin{array}{l}\text { LIPd (fix) } \\
\text { \%Cd }\end{array}$ & $\begin{array}{l}\text { LIPd (fix) } \\
\text { \%Cf }\end{array}$ & $\begin{array}{l}\text { LIPd (all) } \\
\% C d\end{array}$ & $\begin{array}{l}\text { LIPd (all) } \\
\% \text { Cf }\end{array}$ \\
\hline Cd & $48.6 \pm 0.9$ & $48.4 \pm 1.3$ & & & & \\
Cf & $57.5 \pm 1.9$ & $54.8 \pm 3.2$ & $\mathbf{1 8 . 3}$ & & $\mathbf{1 3 . 2}$ & \\
Horizontal & $59.5 \pm 4.3$ & $57.2 \pm 3.9$ & $\mathbf{2 2 . 4}$ & 3.5 & $\mathbf{1 8 . 2}$ & 4.4 \\
Vertical & $55.5 \pm 0.9$ & $54.3 \pm 1.6$ & $\mathbf{1 4 . 2}$ & -3.5 & $\mathbf{1 2 . 2}$ & -0.9 \\
Up & $56.7 \pm 1.9$ & $54.3 \pm 2.8$ & $\mathbf{1 6 . 7}$ & -1.4 & $\mathbf{1 2 . 2}$ & -0.9 \\
Down & $57.9 \pm 1.7$ & $54.5 \pm 3.4$ & $\mathbf{1 9 . 1}$ & 0.7 & $\mathbf{1 2 . 6}$ & -0.5
\end{tabular}

LIPd (fix), LCGU values in the region of LIPd activated for fixation (area specified by (f-Cd effect). LIPd (all), LCGU values in the entire LIPd area. The other conventions are as in Table 2.

experiment, this monkey executed 100-127 saccades to each of the leftward targets $\left(5,10,15\right.$, and $\left.30^{\circ}\right)$ and 191 saccades to the $30^{\circ}$ rightward target (Table 1). Only the right hemisphere of this monkey (contralateral to leftward saccades) was used to study the representation of the horizontal meridian, whereas the left was used to study the representation of $30^{\circ}$ horizontal movements. The second monkey executing saccades along the horizontal meridian (Fig. 1a, monkey $\mathrm{H} 2$ ) was required to perform a sequence of 5,10 , and $15^{\circ}$ movements to the left, followed by a $30^{\circ}$ movement to the right, then a sequence of 5,10 , and $15^{\circ}$ movements to the right, followed by a $30^{\circ}$ movement to the left. It performed $50-90$ saccades to each target during the critical first 10 min of the experiment (Table 1). Both of its hemispheres were used to study the representation of the horizontal meridian in area LIP.

The monkey executing vertical saccades (Fig. $1 b$ ) was required to perform a sequence of 5,10 , and $15^{\circ}$ upward movements, followed by a $30^{\circ}$ downward movement, then a sequence of 5,10 , and $15^{\circ}$ downward movements, followed by a $30^{\circ}$ upward movement. This animal performed 62-105 saccades to each of the upward and downward targets within the critical first $10 \mathrm{~min}$ of the experiment (Table 1), and both of its hemispheres were used to study the representation of the vertical meridian.

Five monkeys (O1-O5) were trained to perform oblique saccades (Fig. $1 c$, Table 1). Two of them $(\mathrm{O} 1, \mathrm{O} 2)$ were trained to execute up-left saccades, 30 and $20^{\circ}$ in amplitude, respectively. During the critical 10 first minutes of the experiment, these monkeys executed 217 and 119 saccades to the peripheral target, respectively. Since both animals had to foveate the fixation point to begin a new trial and since this was always to the right of the target of the previous trial, they would need to execute a combination of spontaneous and visually guided rightward saccades presumably controlled by the left hemisphere. If these were evenly distributed, the left IPs might have been homogeneously activated and could thus have served as a control case in our study. However, the rightward saccades of these two animals were neither concentrated enough in oculomotor space to allow their crisp representation in IPs space nor were they distributed evenly enough for the left IPs of these animals to serve as controls in our study. Thus, only the right hemispheres of $\mathrm{O} 1$ and $\mathrm{O} 2$ monkeys (contralateral to leftward saccades) were used to study the representation of oblique up saccades. For the same reason, only the left hemisphere was used from the third monkey (O3), who had to perform a sequence of two down-right $10^{\circ}$ saccades, and who executed 212 of these during the critical 10 first minutes of the experiment. However, the three

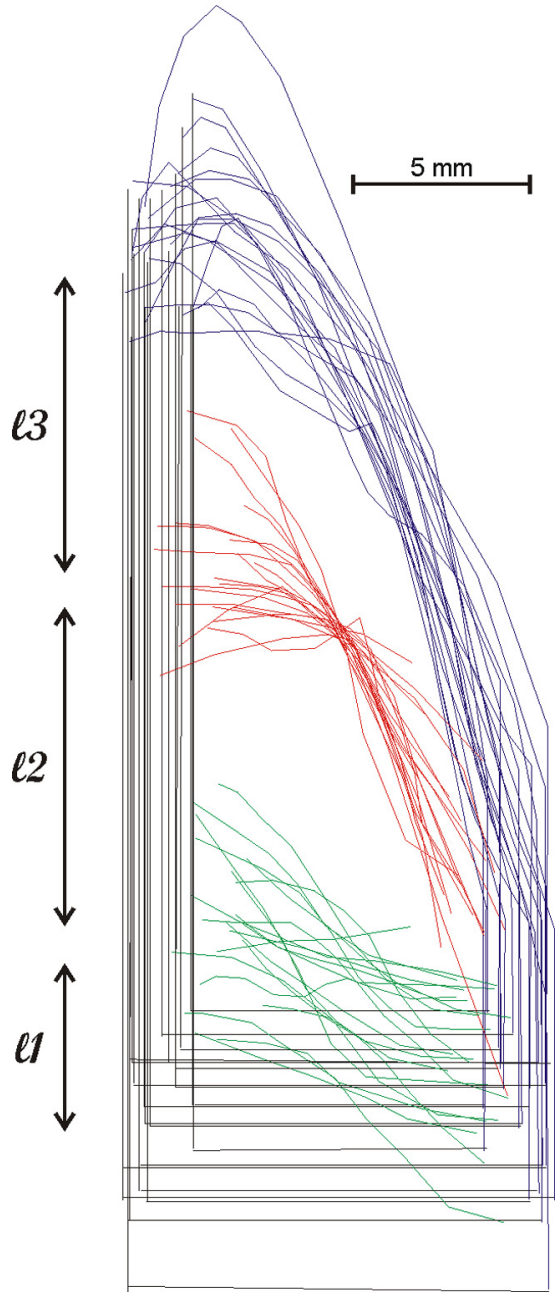

Figure 2. Outlines of the lateral bank of IPs drawn from all of the hemispheres we studied $(N=21)$ and aligned on the center of the LIPv/LIPd border. Outlines from the left hemisphere are reflected to match those from the right. Blue, Crown of the IPs; red, LIPv/LIPd border; green, posterior border of LIPv. The horizontal straight black line indicates the intersection of the IPs with the POs, and the vertical straight black line represents the fundus of the IPs.

hemispheres of monkeys $\mathrm{O} 1, \mathrm{O} 2$, and $\mathrm{O} 3$, ipsilaterally to the executed saccades, allowed us to evaluate and reject the possibility that area LIP might be affected by ipsiversive saccades. Otherwise, these three hemispheres will not be further considered. The fourth monkey (O4) had to execute a sequence of a $20^{\circ}$ up-left saccade followed by two down-right $10^{\circ}$ saccades. During the critical 10 first minutes of the experiment, this monkey performed 130 up-left saccades and 331 down-right saccades and thus contributed one hemisphere contralateral to oblique up, and one contralateral to oblique down saccades. The same was true of another monkey (O5) that was required to perform a sequence of two $10^{\circ}$ up-left saccades followed by one $20^{\circ}$ down-right saccade. During the first $10 \mathrm{~min}$ of the experiment, this animal performed 323 up-left and 156 downright saccades.

In addition to generating $5-30^{\circ}$ visually guided saccades, our monkeys also executed several small $\left(<2^{\circ}\right)$ saccades to the target and fixation points (fixational saccades). Accordingly, and to disambiguate effects caused by large visually guided saccades from those caused by fixation, activation of the IPs of monkeys executing saccades was compared with four hemispheres from two fixation-control monkeys (Cf). These animals were trained to maintain fixation of a central target located straight ahead for the duration of the trial (4s). Intertrial intervals ranged between 0.2 and $0.3 \mathrm{~s}$. The Cf monkeys maintained fixation for $75 \%$ of the $\left[{ }^{14} \mathrm{C}\right] \mathrm{DG}$ experimental time including intertrial intervals. They also executed small fixational saccades that were as numerous as those of the 

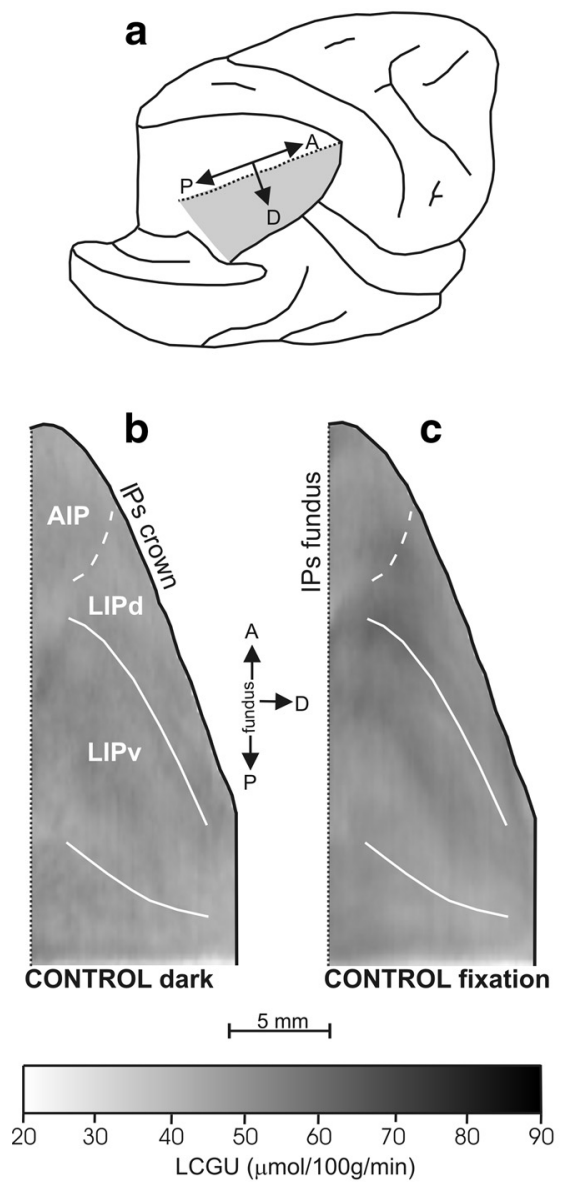

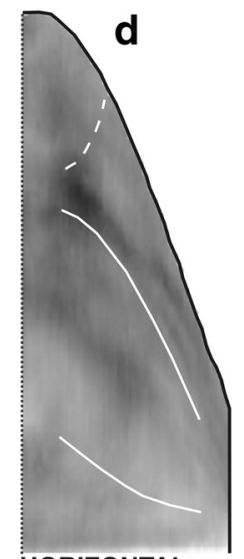

HORIZONTAL

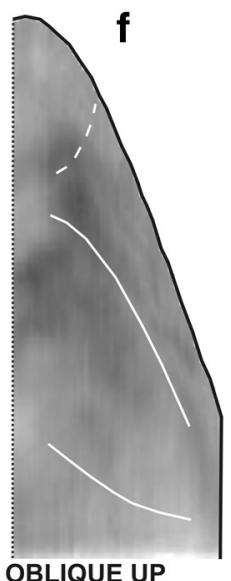

OBLIQUE UP

Figure 3. Two-dimensional reconstructions of the distribution of metabolic activity in area LIP. Quantitative maps of the spatiointensive distribution of $\mathrm{LCGU}$ values (in micromoles $\cdot 100 \mathrm{~g}^{-1} \cdot$ minute ${ }^{-1}$ ) in the lateral bank of the IPs. $\boldsymbol{a}$, Posterolateral view of the partially dissected right hemisphere of a monkey brain. The IPs was unfolded after the inferior parietal lobule was cut away below the posterior (lateral) crown of the IPs, and the occipital lobe was cut away at the fundus of parietoccipital and lunate sulci. The shaded area represents the reconstructed lateral (lower) bank of the IPs. $\boldsymbol{b}$, Two-dimensional map of activity in the cortex of the reconstructed lateral bank of the IPs, averaged from the four hemispheres of the two control monkeys in the dark (Cd). The solid black line on the right represents the crown of IPs; the vertical dotted black line on the left depicts its fundus. The solid white lines correspond to the cytoarchitectonically identified borders of area LIPv. The dashed white line corresponds to the functionally identified border between LIPd and AIP (Evangeliou et al., 2009). c, Two-dimensional map of the lateral bank of IPS, averaged from the four hemispheres of the two control fixating monkeys ( $(f)$. $\boldsymbol{d}$, Averaged map from three hemispheres of monkeys executing contraversive horizontal saccades of 5, 10, 15, and $30^{\circ}$ amplitude.e, Averaged map from two hemispheres of the monkey executing vertical saccades of 5, 10, 15, and $30^{\circ}$ amplitude up and down. $f$, Averaged map from four hemispheres of monkeys executing contraversive saccades of 10,20 , and $30^{\circ}$ amplitude from straight ahead in an oblique direction $135^{\circ}$ up. $\boldsymbol{g}$, Averaged map from three hemispheres of monkeys executing contraversive saccades of 10 and $20^{\circ}$ amplitude from straight ahead in an oblique direction $315^{\circ}$ down. Gray-scale bar indicates LCGU values in micromoles $\cdot 100 \mathrm{~g}^{-1} \cdot$ minute ${ }^{-1}$. A, Anterior; AIP, anterior intraparietal area; $D$, dorsal; $P$, posterior.

other monkeys, and some large spontaneous saccades spread throughout their oculomotor range. Thus, in addition to removing visual effects caused by fixation, subtraction of metabolic effects found in the Cf monkeys from those of the monkeys executing saccades removes effects caused by fixational saccades. To reveal effects induced by fixation, glucose utilization values obtained from the Cf monkeys were compared with those obtained from four hemispheres from two monkeys (Cd) that were seated in the dark in front of the idle apparatus for the duration of the experiment. To achieve darkness, the primate chair was enclosed within black curtains together with the idle video monitor. Reward was delivered at random intervals, so that the total number of rewards matched that of monkeys rewarded for executing visually guided saccades. These monkeys were alert during the whole $45 \mathrm{~min}$ of the $\left[{ }^{14} \mathrm{C}\right] \mathrm{DG}$ experiment as demonstrated by the execution of spontaneous saccades evenly spread throughout the oculomotor range of the animals.

Quantitative cortical maps. The $\left[{ }^{14} \mathrm{C}\right] \mathrm{DG}$ experiments, brain sectioning, and quantitative autoradiography were performed as previously de-

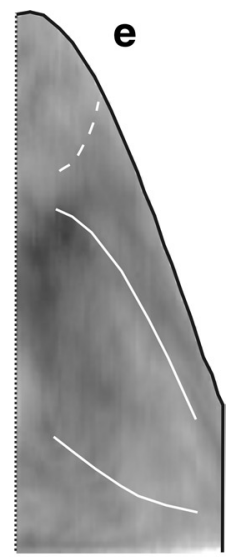

\section{VERTICAL}

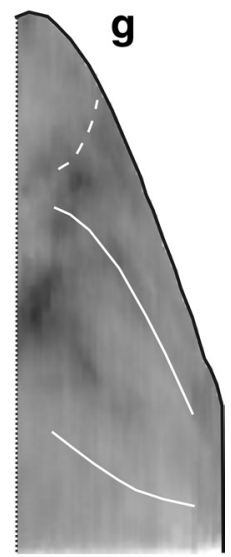

OBLIQUE DOWN scribed (Gregoriou and Savaki, 2001). The metabolic activity in local cerebral glucose utilization (LCGU) values (in micromoles $100 \mathrm{~g}^{-1}$. minute ${ }^{-1}$ ) was calculated from the original operational equation of the method (Sokoloff et al., 1977) using the appropriate kinetic constants for the monkey (Kennedy et al., 1978). The full extent of the IPs was analyzed in both hemispheres of each animal, each extending through $\sim 550$ serial horizontal sections of $20 \mu \mathrm{m}$ thickness. One section of every 25 was stained with thionin for cytoarchitectonic identification of areas LIP ventral (LIPv) and LIP dorsal (LIPd) within area 7 of the lateral bank of the IPs, according to the criteria of Medalla and Barbas (2006). The spatial distribution of metabolic activity within the rostrocaudal and dorsoventral extent of each IPS was reconstructed in two-dimensional maps as previously described (Savaki et al., 1997; Bakola et al., 2006). Briefly, for each horizontal, $20-\mu \mathrm{m}$-thick, section through the IPs, a data array was generated by sampling LCGU values (at a resolution of 50 $\mu \mathrm{m} /$ pixel) along a line that ran parallel to the surface of the cortex and whose thickness was equal to that of the cortical gray matter. LCGU values were averaged every five adjacent sections to avoid cutting artifacts. Accordingly, each two-dimensional reconstruction of the IPs is made of 110 lines (550 sections divided by five sections per line), and each line represents the average of five adjacent serial sections. The dorsoventral plotting resolution of our two-dimensional maps of the IPs thus equals $100 \mu \mathrm{m}$, a resolution we also adopted for the anteroposterior axis of the same maps. The intersection of the IPs with the parieto-occipital sulcus (POs) was used for the alignment of adjacent data arrays (Bakola et al., 2006). To normalize metabolic activity (Bakola et al., 2006), LCGU values were multiplied with a factor that was separately determined for each hemisphere. This factor is equal to the ratio of the mean LCGU value found in the unaffected medial bank of the IPs of the hemisphere in question over the mean LCGU value obtained from the same bank after pooling all hemispheres from all monkeys. Very similar normalization factors were found when LCGU values from other unaffected cortical areas (such as the limb and body representations of the primary motor and somatosensory cortices in the central sulcus) were used instead. Percentage LCGU differences between experimental and control subjects were calculated as follows: (experimental - control)/control ${ }^{\star} 100$. To determine statistical significance (Tables 2, 3, bold), we relied on Student's unpaired $t$ test (Bakola et al., 2007).

The size of the IPs, the length and the depth of area LIP, as well as the precise location of the LIPv/LIPd border and therefore the size of LIPv and LIPd varied considerably from animal to animal (Fig. 2). To compensate for this variability, individual two-dimensional functional reconstructions of LIPv and LIPd were geometrically normalized together with their cytoarchitectonically identified borders, thus allowing the direct comparison of the sites of activation. In each horizontal section, we measured the distances between the intersection of the IPs with the POs and the posterior border of $\operatorname{LIPv}\left(l_{1}\right)$ (Fig. 2, green), between the posterior and the anterior border of $\operatorname{LIPv}\left(l_{2}\right)$ (Fig. 2, red), and between the anterior border of LIPv and the crown of the IPs $\left(l_{3}\right)$ (Fig. 2, blue). Averages of these lengths were separately estimated from all of the hemi- 

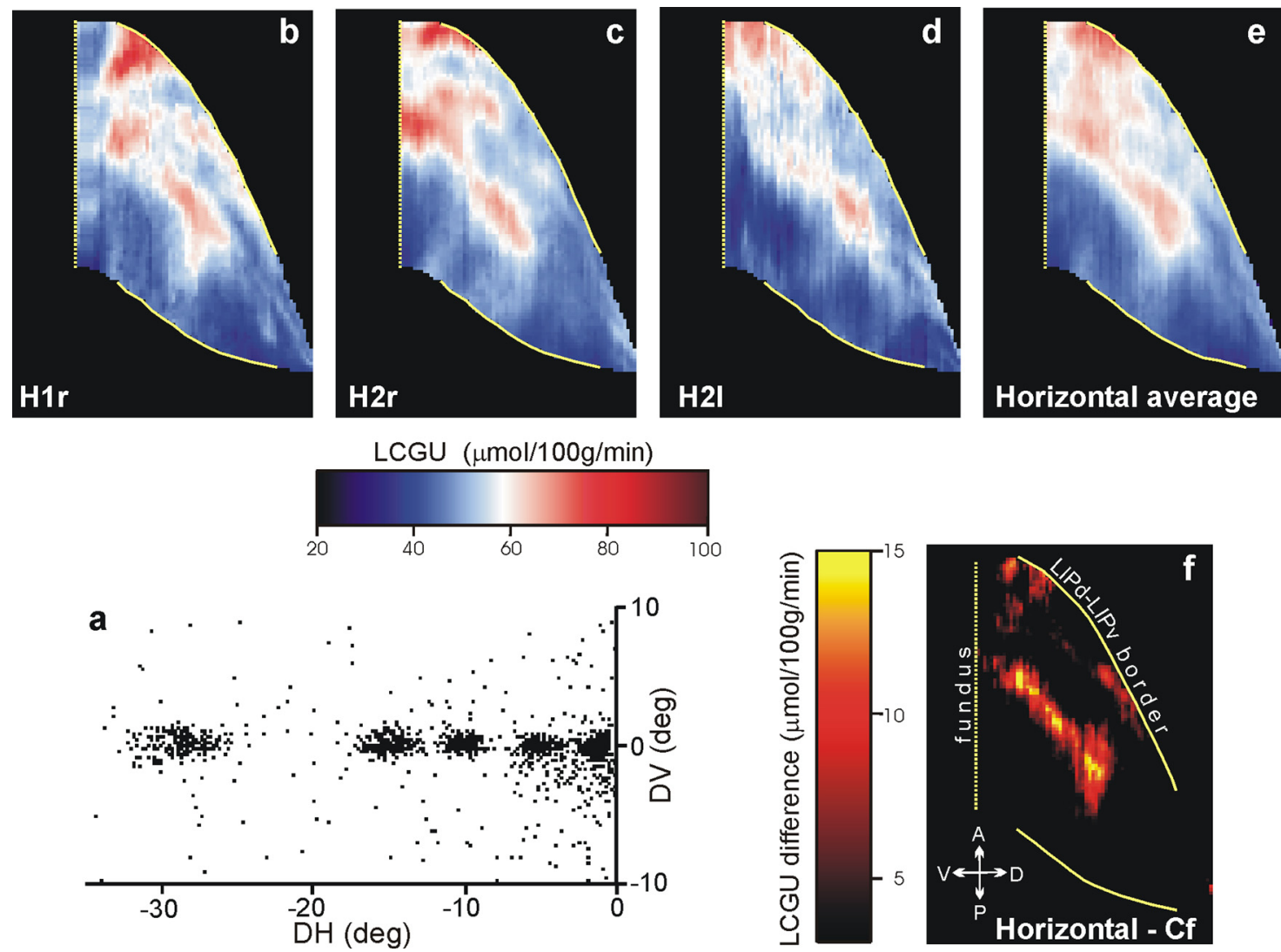

Figure 4. Representation of the horizontal meridian in area LIPv. $\boldsymbol{a}$, Cumulative scatterplot of the endpoints of all saccade vectors (plotted relative to eye position at the start of each saccade) executed by two monkeys during the critical first $10 \mathrm{~min}$ of the $\left[{ }^{14} \mathrm{C}\right] \mathrm{DG}$ experiment to visual targets lying along a straight horizontal line through the primary eye position, 5 , 10,15 , and $30^{\circ}$ away from straight ahead. $\boldsymbol{b}$ - $\boldsymbol{d}$, Rightward saccades of monkey $\mathrm{H} 2$ were reflected and are shown as leftward. Individual quantitative two-dimensional maps of the distribution of metabolic activity in area LIPv in the right hemisphere of monkey $\mathrm{H} 1$ ( $\mathrm{H} 1 \mathrm{r}$ ) and both hemispheres of monkey $\mathrm{H} 2$ ( $\mathrm{H} 2 \mathrm{r}, \mathrm{H} 2 \mathrm{l})$. $\boldsymbol{e}$, Two-dimensional map of metabolic activity in area LIPv averaged over the three hemispheres illustrated in $\boldsymbol{b}$ - $\boldsymbol{d}$. $\boldsymbol{f}$, Two-dimensional reconstruction of percentage differences in LIPv obtained from the map in $\boldsymbol{e}$ after subtracting the corresponding activity in the fixating control ( $\mathrm{C} f$ ) monkeys. The location and orientation of LIPv relative to other portions of the lateral bank of the IPs is shown in Figure 3 . The vertical straight yellow dotted line, on the left, represents the fundus of the IPs. The solid yellow lines correspond to the cytoarchitectonically identified borders of area LIPv. The red/white/blue color bar indicates LCGU values in micromoles $\cdot 100 \mathrm{~g}^{-1} \cdot$ minute $^{-1}$. The yellow/red color bar indicates $\mathrm{LCGU}$ difference from the fixation control in micromoles $\cdot 100 \mathrm{~g}^{-1} \cdot$ minute $^{-1}$. A, Anterior; D, dorsal; $\mathrm{P}$, posterior; $\checkmark$, ventral.

spheres to construct a reference surface landmark map. The twodimensional reconstruction of each hemisphere was then fit to this reference map using linear transformations of the plane (Moschovakis et al., 1990, 2004) with the help of custom-designed routines in the Matlab environment (Mathworks). With this procedure, we created a geometrically normalized map of area LIP containing a standard number of pixels. Data from different geometrically normalized maps were combined to obtain the average- and difference-LCGU maps we illustrate. To generate average maps, the LCGU value found in a certain pixel in one of the geometrically normalized maps was added to the value found in the pixel occupying the same position in one or more other similar maps and the result was divided by the number of maps used. Similarly, to generate a difference map, the LCGU value found in a certain pixel of a geometrically normalized map of a control hemisphere was subtracted from the value found in the pixel occupying the same position in a similar map obtained from an experimental animal.

\section{Results}

Area LIP has been subdivided into a dorsal and a ventral part on the basis of neuroanatomical criteria. Its dorsal part (LIPd) is located superficially (closer to the crown), whereas the ventral (LIPv) is more extensive and lies closer to the fundus. LIPv is heavily myelinated (Medalla and Barbas, 2006) and its connections with the FEF and the SC are stronger than those of LIPd (Lynch et al., 1985; Blatt et al., 1990; Schall et al., 1995). Since area
LIPv harbors the representation of oculomotor space in area 7IP (Bakola et al., 2006), the former is the primary object of this study.

We found its cytoarchitectonic boundaries in sections stained for Nissl following the criteria previously established by others (Medalla and Barbas, 2006). We reconstructed the cytoarchitectonically identified borders in two-dimensional anatomical maps, in a manner similar to the one we used for the twodimensional functional maps (see Materials and Methods, Quantitative cortical maps), so that functional and anatomical reconstructions could be superimposed. Because area LIP is known to represent the contralateral one-half of visual space (Gnadt and Andersen, 1988; Blatt et al., 1990), and its microstimulation to evoke contraversive saccades (Mushiake et al., 1999), we report only saccades contraversive to the reconstructed IPs. Figure 3 shows quantitative two-dimensional maps of the spatiointensive distribution of metabolic activity (in micromoles $100 \mathrm{~g}^{-1} \cdot$ minute $^{-1}$ of glucose consumption) within the lateral bank of the IPs of control monkeys (Cd and Cf) and of monkeys executing saccades in different directions (horizontal, vertical, oblique up or down), along with the cytoarchitectonically identified borders of LIPv. Each map is from a separate group of hemispheres and illustrates the spatial distribution of 


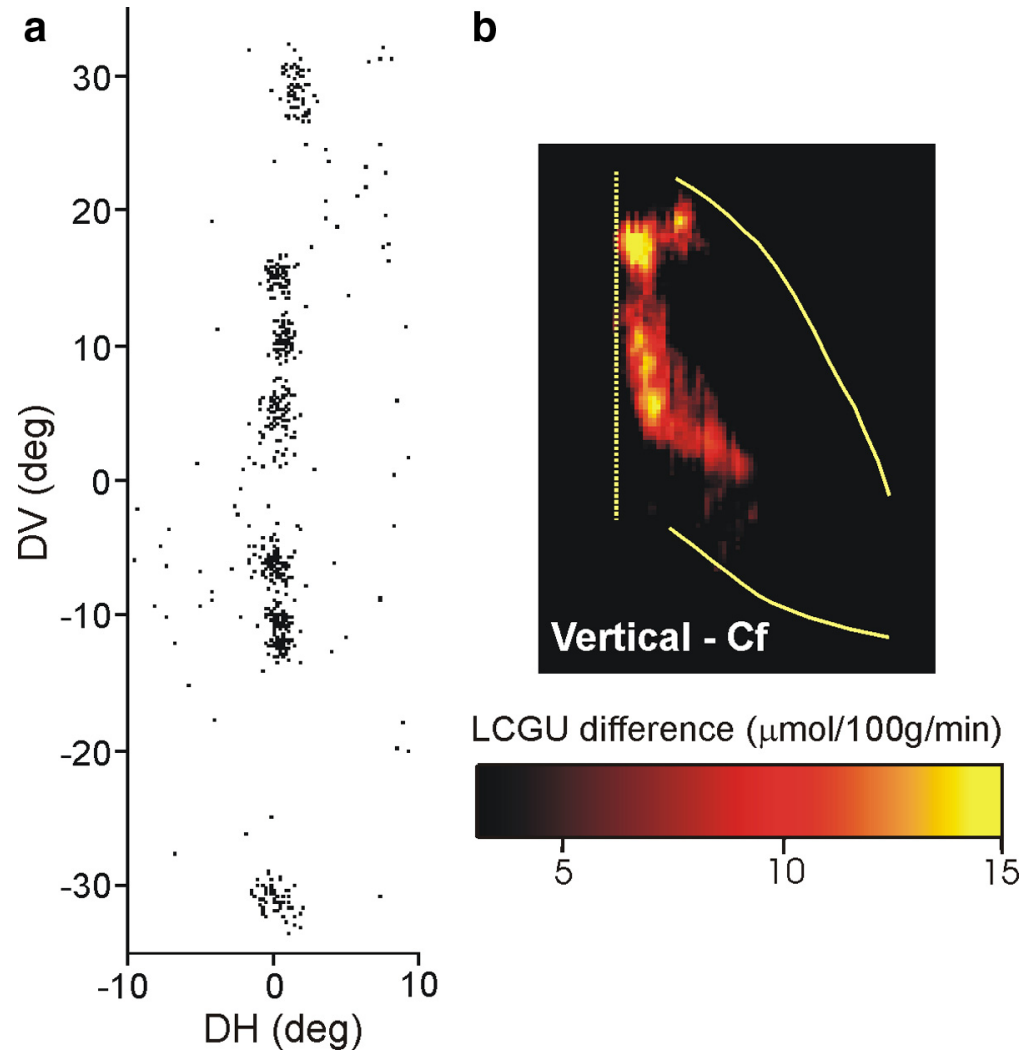

Figure 5. Representation of the vertical meridian in LIPv. $\boldsymbol{a}$, Scatterplot of the endpoints of all saccade vectors (plotted relative to eye position at the start of each saccade) executed by a monkey during the critical first $10 \mathrm{~min}$ of the $\left[{ }^{14} \mathrm{C}\right] \mathrm{DG}$ experiment to visual targets located on a vertical line $5,10,15$, and $30^{\circ}$ away from straight ahead. $\boldsymbol{b}$, Quantitative two-dimensional map of activity in area LIPv averaged over both hemispheres, after subtracting the map of activity averaged over the same area in the Cf monkeys. Layout is as in Figure 4.

activity in a geometrically normalized map of the lateral bank of the IPs averaged across all members of the group. In this and the following figures, reconstructions from the left hemisphere are reflected to match those from the right, whereas anterior is up and posterior is down.

Figure $4 a$ is the cumulative scatterplot of the endpoints of the vectors of all horizontal visually guided saccades executed by monkey $\mathrm{H} 2$ and the leftward ones executed by monkey $\mathrm{H} 1$ in the critical 10 first minutes of the experiment. Because these were executed in sequences of saccades measuring $5,10,15$, and $30^{\circ}$ toward a series of appropriately spaced targets, the three hemispheres contralateral to the movements might allow us to discern the representation of the horizontal meridian. Figure $4 b-d$ illustrates two-dimensional reconstructions of the metabolic activity found in the LIPv of individual hemispheres, and Figure $4 e$ is their average. As shown here, the horizontal meridian is represented as an almost contiguous zone of increased metabolic activity (redyellow) running through the center of the LIPv, parallel to its border with LIPd. This pattern of activation becomes even more evident (Fig. $4 f$ ) after subtracting the pattern of activation in the $\mathrm{LIPv}$ of the two control animals (Cf) that were rewarded for maintaining fixation of a target presented straight ahead (Fig. 3c). The representation of the $30^{\circ}$ rightward horizontal saccades in the left hemisphere of $\mathrm{H} 1$ occupied part of the representation of the horizontal meridian in area LIPv.

Similarly, to visualize the representation of the vertical meridian in the LIPv, another rhesus monkey performed 5, 10, 15, and $30^{\circ}$ upward and downward visually guided saccades. Figure $5 a$ is the cumulative scatterplot of the endpoints of the vectors of all vertical saccades generated by this animal in the critical 10 first minutes of the experiment. The two-dimensional reconstruction of the average metabolic activity pattern in the lateral bank of IPs of the two hemispheres of this animal is shown in Figure $3 e$. The pattern of activation in its LIPv after subtracting the average pattern of activation in the two Cf control monkeys (Fig. 3c) displays an almost contiguous zone of increased metabolic activity (red-yellow) running parallel to the fundus of the IPs (Fig. 5b).

Figure $6 a$ illustrates the cumulative scatterplot of the endpoints of saccades executed by four animals that were rewarded for performing up-left oblique saccades: the first one (O1) to a target $30^{\circ}$ away from straight ahead in an oblique direction $45^{\circ}$ up and to the left, two others (O2 and $\mathrm{O} 4$ ) to a target $20^{\circ}$, and the remaining one (O5) to a target $10^{\circ}$ away from straight ahead in the same up-left direction. The two-dimensional distribution of metabolic activity in the lateral bank of the IPs of their right hemispheres, averaged over all four subjects, is shown in Figure $3 f$. Subtraction of the LIPv map averaged over the four hemispheres of the Cf control animals from the LIPv map averaged over the four hemispheres of animals performing up-left oblique saccades demonstrates that oblique upward saccades increased metabolic activity in the rostral LIPv toward its border with the LIPd (Fig. 6b).

In turn, oblique downward saccades would be expected to activate a region in the contralateral LIPv caudal to the representations of the horizontal meridian toward the caudal borders of the IPs. This is indeed the case as shown by the average twodimensional reconstruction of the spatial distribution of metabolic activity in the left LIPv of three animals that executed down-right saccades, before (Fig. $3 g$ ) and after (Fig. 6c) subtraction of the corresponding Cf map. As illustrated in Figure $6 d$, one of these monkeys (O5) executed saccades to a target $20^{\circ}$ away from straight ahead in an oblique direction $45^{\circ}$ down and to the right, and two other monkeys (O3 and $\mathrm{O} 4$ ) to a target in the same down-right direction but $10^{\circ}$ away from straight ahead.

To explore whether neural space is also used to represent saccade size in the IPs, we compared the activation found in two animals (O2 and $\mathrm{O} 4)$ for upward saccades of $\sim 20^{\circ}$ to that for bigger upward saccades $\left(\sim 30^{\circ}\right)$ found in another animal $(\mathrm{O} 1)$ as well as to that for upward saccades of $\sim 10^{\circ}$ in a fourth animal (O5). Figure $7 a$ illustrates the average endpoint of all upward saccades executed toward the targets by these four animals $\left(10^{\circ}\right.$, triangle; $20^{\circ}$, circle and diamond; $30^{\circ}$, square) during the critical 10 first minutes of the $\left[{ }^{14} \mathrm{C}\right] \mathrm{DG}$ experiment. We found saccades of large amplitude $\left(30^{\circ}\right)$ represented close to the crown (Fig. $\left.7 b\right)$, smaller ones $\left(10^{\circ}\right)$ close to the fundus (Fig. $7 d$ ), whereas saccades of intermediate size $\left(20^{\circ}\right)$ are represented at intermediate sites in the $\operatorname{LIPv}$ (Fig. 7 c).

LCGU values measured in LIPv subregions representing the horizontal meridian, the vertical meridian, oblique upward, and oblique downward saccades (shown in Figs. 4-6) are separately reported in Table 2 . Because the number of saccades toward a 
target was not the same in all animals ( $\mathrm{Ta}$ ble 1) nor was the intensity of the saccaderelated activation in LIPv the same in all hemispheres, we examined whether LIPv activation is correlated with the number of saccades executed. To this end, we estimated the percentage LCGU difference (\%Dif) between the activated and surrounding areas of LIPv separately for each of the hemispheres we examined. For each monkey, we defined the activated LIPv region as the set of pixels whose values surpassed the mean LIPv activation by at least $1 \mathrm{SD}$ and expressed their average activation $(A)$ as the mean of their LCGU values. We also estimated the average background activity of surrounding areas $(B)$ as the mean of the LCGU values of the remaining pixels (i.e., those with LCGU values smaller than the mean $+1 \mathrm{SD}$ of the mean) and expressed the \%Dif between the activated and surrounding areas of LIPv as $100^{*}(A-B) / B$. A plot of LIPv $\%$ Dif against the number of contraversive, visually guided saccades performed during the critical 10 first minutes of the experiment indicates a statistically significant correlation between the two variables $(r=0.62 ; p<0.05)$. However, LIPv $\%$ Dif was even better correlated $(r=0.77$; $p<0.005$ ) with saccade density (defined as the number of saccades toward a target divided by the area they collectively occupy), such that in this case the independent variable accounts for $59 \%$ of the variance of the dependent variable (Fig. 8 ). This should not come as a surprise if the representation of saccades in area LIP is topographically organized. Conceivably, diffusely dispersed saccades could be represented in a larger, albeit less intensely activated, area than that representing movements focused in space. The slope of the relationship is rather shallow demonstrating that a more than ninefold (>900\%) difference in behavior (the range of saccades/ $\left.\mathrm{deg}^{2}, 2-19\right)$ leads to differences in glucose consumption $<50 \%$ (the range of percentage LCGU difference, 23-33).

As expected from our previous observations (Gregoriou and Savaki, 2001; Bakola et al., 2006), the LIPd of our monkeys was also activated (Fig. 9, Table 3), but in this case its intensity depended on the presence of visual targets and their fixation. To reveal the pattern of fixation-related activation in the IPs, we had to use animals other than the fixating ones (Cf) as a control. Here, we used the Cd monkeys instead. When the map of LCGU values obtained from the Cd monkeys was subtracted from the map of the Cf monkeys, the resulting fixation-related activation was found at the border of LIPd and LIPv extending mainly in LIPd (Fig. $9 e$, Cf-Cd). The same region of LIPd was found activated when the functional maps of Cd monkeys were subtracted from those of monkeys executing horizontal, vertical, oblique up, and oblique down saccades (Fig. $9 a-d$, respectively). In contrast, little of area LIPd remained activated when functional maps of Cf monkeys were subtracted from those of monkeys executing horizontal, verti- cal, oblique up, and oblique down saccades (Fig. $9 f-i$, respectively), thus confirming our previous observation that LIPd activation depends on the presence of visual targets and their fixation (Bakola et al., 2006).

\section{Discussion}

The present report is the first to provide high-resolution quantitative functional images of the representation of saccade metrics in the lateral bank of the IPs. Our major findings are summarized in Figure 10, which illustrates geometrically normalized LIPv maps from all 21 of the hemispheres we studied. As shown in Figure $10 a$, the representation of the vertical meridian (in blue) runs mostly parallel to the fundus of the IPs. Rather than being orthogonal to it, the representation of the horizontal meridian (red) runs through the middle of LIPv parallel to its border with LIPd. Also, oblique downward saccades (violet) are represented in a region of the LIPv that lies behind the representation of the horizontal meridian toward the caudal edge of the IPs, whereas oblique upward saccades (green) are represented in front of the representation of the horizontal meridian toward the border be- 

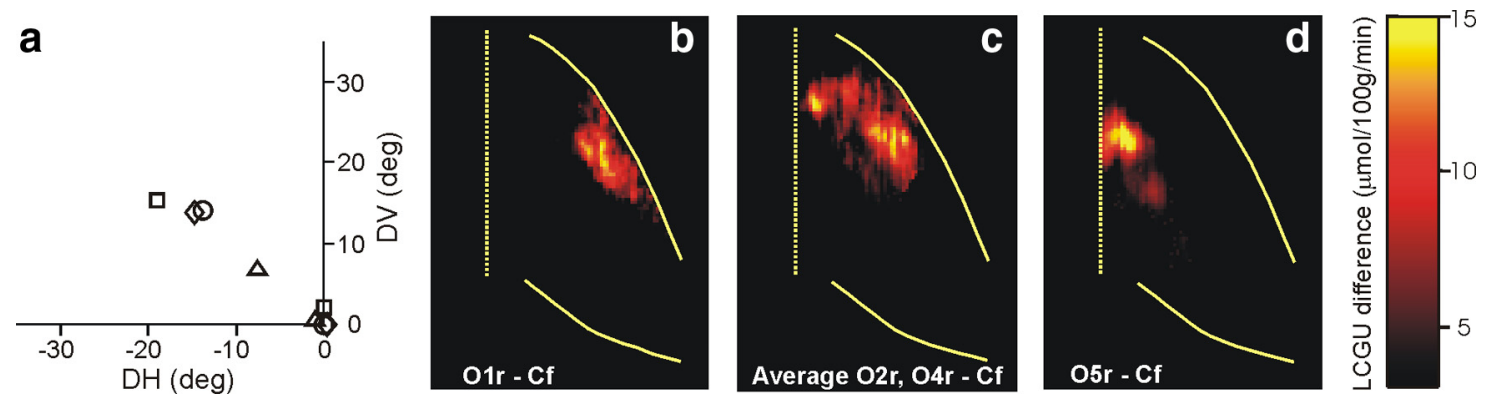

Figure 7. Quantitative maps of activity in area LIPv accompanying oblique up-left saccades of three different sizes. $\boldsymbol{a}$, Average location of the endpoints of all saccade vectors (plotted relative to eye position at the start of each saccade) executed during the critical first $10 \mathrm{~min}$ of the $\left[{ }^{14} \mathrm{C}\right] \mathrm{DG}$ experiment. Each symbol is from a different animal. Triangle, $10^{\circ}$ saccades; circle and diamond, $20^{\circ}$ saccades; square, $30^{\circ}$ saccades. $\boldsymbol{b}$, Reconstructed two-dimensional map of activity in the right LIPv of the animal executing $30^{\circ}$ saccades up-left, after subtracting the Cf control map. c, Two-dimensional map of the average activity in the right LIPv of the two monkeys executing $20^{\circ}$ saccades up-left (minus the (f control map). $\boldsymbol{d}$, Reconstructed two-dimensional map of activity in the right LIPv of the animal executing $10^{\circ}$ saccades up-left (minus the (f control map).

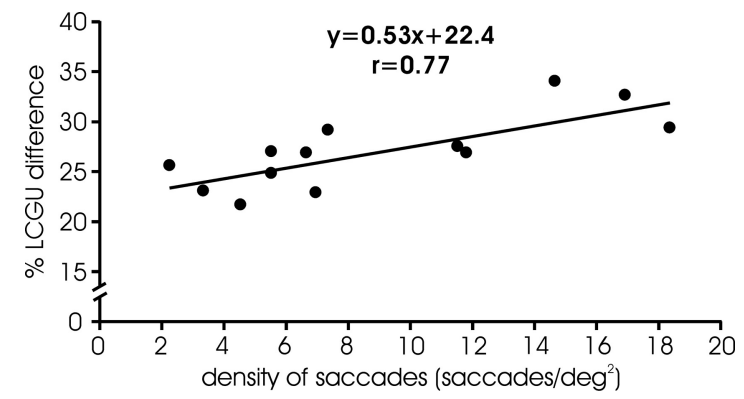

Figure 8. Relationship between saccade density and glucose consumption in LIPv. Plot of percentage (\%) LCGU difference between the LIPv region that was activated and the tissue surrounding the activation (ordinate) against the density of contraversive visually guided saccades (abscissa). Each data point is from a different hemisphere. The solid line is the linear regression line through the data and obeys the equation displayed.

tween LIPv and LIPd. Finally, as shown in Figure 10b, size is also represented in an orderly manner in the LIPv, at least in the region devoted to upward saccades, which is extensive enough to be explored with our method. The smallest saccades (red) are represented close to the fundus of the IPs near the intersection of the horizontal with the vertical meridian, bigger saccades (green) are represented toward the crown of the sulcus, and saccades of intermediate size (blue) are represented in between.

To our knowledge, this study is the first to demonstrate that saccade metrics are topographically organized in area LIP of the monkey and, in particular, its ventral portion (area LIPv). Previous attempts to establish the topographic organization of area LIP and its relationship to the representation of space did not focus on LIPv and relied on visual rather than motor-related neuronal responses or properties. For example, the encounter frequency of neurons with central visual field was found to increase rostrally and that of neurons with peripheral visual field to increase caudally in area LIP (Ben Hamed et al., 2001). These data can be interpreted in light of the method we used, which allowed us to discern the cytoarchitectonic boundaries of the intraparietal areas that were activated. Judging from these, it is tempting to speculate that the central visual field representation of Ben Hamed et al. (2001) extends into area LIPd of the present study, whereas their representation of peripheral visual stimuli occupies much of our LIPv. Past efforts to explore the representation of space in the IPs also relied on functional magnetic resonance imaging techniques in humans (Sereno et al., 2001; Silver et al., 2005; Swisher et al., 2007). However, the data reported herein appear to be much more orderly and repeatable than any results so far reported in humans. To a large extent, this is attributable to the advantages of the $\left[{ }^{14} \mathrm{C}\right] \mathrm{DG}$ method: (1) direct assessment of brain activity (in glucose consumption), rather than the indirect estimates obtained from other imaging techniques (e.g., via blood flow); (2) quantitative measurement of local cerebral glucose utilization, instead of the qualitative or semiquantitative estimates provided by other brain imaging methods; (3) plotting resolution of $100 \mu \mathrm{m}$ (i.e., spatial resolution one order of magnitude or more than other imaging methods); and (4) cytoarchitectonic identification of cortical areas in sections adjacent to the autoradiographic ones, allowing the accurate anatomical localization of functional activations.

The relative sizes and locations of LIPd and LIPv in our maps are mostly in agreement with previous descriptions (Lewis and Van Essen, 2000; Medalla and Barbas, 2006). For example, the LIPv of Medalla and Barbas (2006) occupies approximately the deep one-half of the lateral intraparietal bank, whereas area LIPd is located more superficially and occupies approximately onefourth to one-third of the bank. Also, the reconstructed maps in the study by Lewis and Van Essen (2000), their Figures 4b, 11 $a-d$, 14 , and 15, illustrate the LIPd-LIPv border at an acute angle relative to the fundus of the IPs. There are, however, striking differences in shape. LIPv has been previously presented as a strip-like structure [Lewis and Van Essen (2000), their Fig. 11], whereas in our maps it is triangular or wedge-like, narrow at both anterior and posterior ends and thick in the middle. These discrepancies are most probably attributable to methodological differences. The maps of Lewis and Van Essen (2000) result from the tessellation of the cortex and are based on immunohistochemistry and the myelination patterns observed in frontal sections, whereas ours are based on cytoarchitectonic criteria and result from the unfolding (anteroposteriorly) and projection (dorsoventrally) of the IPs onto the sagittal plane. In the plane of sectioning that we used, we could reliably identify the cytoarchitectonic border between LIPd and LIPv. The same is true of the border of 7a with LIPd at the lateral crown of the IPs, as shown in one of our previous studies (Bakola et al., 2006). To our knowledge, no cytoarchitectonic definition of the ventral intraparietal area (VIP) has been published to date. Instead, descriptions of the borders of area VIP are based on myelin staining or SMI-32 immunohistochemistry. Unfortunately, these staining methods require perfused and fixed preparations and are thus not compatible with the $\left[{ }^{14} \mathrm{C}\right] \mathrm{DG}$ method. Accordingly, the representation of the vertical meridian could extend into area VIP. This is consistent with the fact that saccade zones extend toward the 

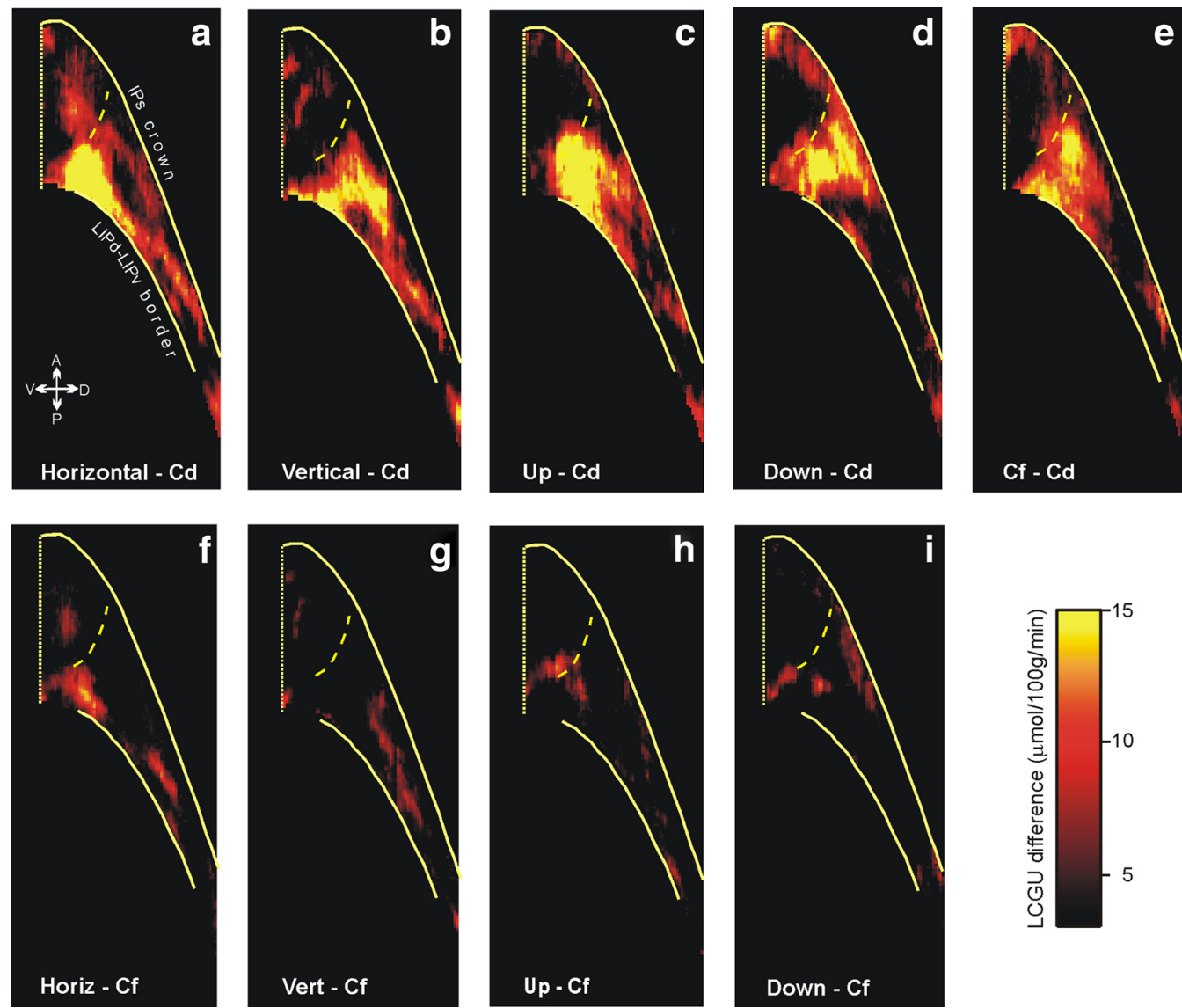

Figure 9. Effects in LPd, expressed as LCGU differences relative to Cd and to (f monkeys. $\boldsymbol{a}$, Quantitative two-dimensional map of activity in the LIPd of monkeys executing horizontal saccades, averaged over the three hemispheres of monkeys $\mathrm{H} 1$ and H2 shown in Figure 4, after subtracting the average of the four hemispheres of the Cd monkeys. $\boldsymbol{b}$, Reconstructed two-dimensional map of activity in the LPd averaged over both hemispheres of a monkey executing vertical saccades, minus the C control map. $c$, Two-dimensional map of the average activity in the LIPd of four monkeys executing oblique upward saccades averaged over all four contralateral hemispheres, minus the Cd control map. $\boldsymbol{d}$, Reconstructed two-dimensional map of the average activity in the LIPd of threemonkeys executing oblique downward saccades, averaged over all three contralateral hemispheres, minustheCd control map.e, Reconstructed two-dimensional map of theactivityin the LPd offfmonkeys averaged overfourhemispheres, minus theCd control map.f, Quantitativetwo-dimensional map of activityin the LIPd of monkeys executing horizontal saccades, after subtracting the average of the fourhemispheres of the (f monkeys. $\boldsymbol{g}$, Reconstructed two-dimensional map of activity in the LIPd averaged over both hemispheres of a monkey executing vertical saccades, minus the Cf map. $\boldsymbol{h}$, Two-dimensional map of the average activity in the LIPd of four monkeys executing oblique upward saccades, minus the (f map.i, Reconstructed two-dimensional map of the average activity in the LIPd of three monkeys executing oblique downward saccades, minus the (ffmap. The color bar indicates LCGU differences from the control (Cd or Cf) in micromoles $\cdot 100 \mathrm{~g}{ }^{-1} \cdot$ minute ${ }^{-1}$. The location and orientation of LIPd relative to other portions of the lateral bank of the IPsis shown in Figure4. The vertical straightyellow line on the left is the fundus of the IPS. The upper solid yellow line on the rightis the crown of the IPS, the lower solid yellow line is the cytoarchitectonically identified border between LPv and LIPd, and the dashed yellow line is the functionally identified border between LIPd and AIP (Evangeliou et al., 2009).
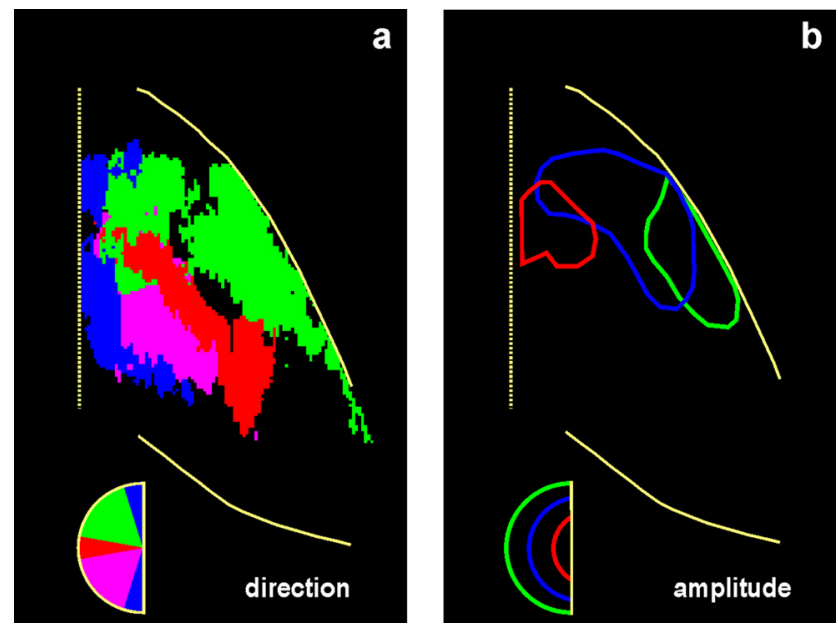

Figure 10. Maps summarizing the topography ofsaccade metricsin area LIPv. $\boldsymbol{a}$, Effects of direction. To be given a certain color, a pixel had to display activation significantly higher than that of the same pixel in theaveraged geometricallynormalized (fmap forsaccadesin oneoffourdirections:vertical(blue), horizontal (red), oblique up (green), or oblique down (violet). Note that this map carries no information regarding the intensity of effects. $\boldsymbol{b}$, Effects of amplitude. Outlines of the main effects of three sizes of upward saccades are illustrated: small ( $\sim 10^{\circ}$; red), big $\left(\sim 30^{\circ}\right.$; green), and intermediate $\left(\sim 20^{\circ}\right.$; blue). fundus of the IPs sharing territory with area VIP (Thier and Andersen, 1998).

In conclusion, the present study demonstrates that the representation of saccade metrics is topographically organized in the primate LIP. The vertical meridian is represented along a line running mostly parallel to the fundus of the IPs, the horizontal meridian along a line that runs through the middle of LIPv and approximately parallel to its borders with LIPd, the upward part of oculomotor space is represented in front of the representation of the horizontal meridian toward the border between LIPv and LIPd, and the downward part of oculomotor space in a region of the LIPv that lies behind the representation of the horizontal meridian toward the caudal edge of the IPs. Small-amplitude upward saccades are represented at the intersection of horizontal and vertical meridians toward the fundus of IPs, whereas bigger upward saccades are represented toward the crown of the IPs.

\section{References}

Bakola S, Gregoriou GG, Moschovakis AK, Savaki HE (2006) Functional imaging of the intraparietal cortex during saccades to visual and memorized targets. Neuroimage 31:1637-1649.

Bakola S, Gregoriou GG, Moschovakis AK, Raos V, Savaki HE (2007) Saccade- 
related information in the superior temporal motion complex: quantitative functional mapping in the monkey. J Neurosci 28:2224-2229.

Ben Hamed S, Duhamel JR, Bremmer F, Graf W (2001) Representation of the visual field in the lateral intraparietal area of macaque monkeys: a quantitative receptive field analysis. Exp Brain Res 140:127-144.

Blatt GJ, Andersen RA, Stoner GR (1990) Visual receptive field organization and cortico-cortical connections of the lateral intraparietal area (area LIP) in the macaque. J Comp Neurol 299:421-445.

Evangeliou MN, Raos V, Galletti C, Savaki HE (2009) Functional imaging of the parietal cortex during action execution and observation. Cereb Cortex 19:624-639.

Gnadt JW, Andersen RA (1988) Memory related motor planning activity in posterior parietal cortex of macaque. Exp Brain Res 70:216-220.

Gregoriou GG, Savaki HE (2001) The intraparietal cortex: subregions involved in fixation, saccades and in the visual and somatosensory guidance of reaching. J Cereb Blood Flow Metab 21:671-682.

Kennedy C, Sakurada O, Shinohara M, Jehle J, Sokoloff L (1978) Local cerebral glucose utilization in the normal conscious macaque monkey. Ann Neurol 4:293-301.

Lewis JW, Van Essen DC (2000) Mapping of architectonic subdivisions in the macaque monkey, with emphasis on parieto-occipital cortex. J Comp Neurol 428:79-111.

Lynch JC, Graybiel AM, Lobeck LJ (1985) The differential projection of two cytoarchitectonic subregions of the inferior parietal lobule of macaque on the deep layers of the superior colliculus. J Comp Neurol 235:241-254.

Medalla M, Barbas H (2006) Diversity of laminar connections linking periarcuate and lateral intraparietal areas depends on cortical structure. Eur J Neurosci 23:161-179.

Moschovakis AK, Scudder CA, Highstein SM (1990) A morphological basis for Hering's law: projections to extraocular motoneurons. Science 248:1118-1119.

Moschovakis AK, Scudder CA, Highstein SM (1996) The microscopic anatomy and physiology of the mammalian saccadic system. Progr Neurobiol 50:133-254.

Moschovakis AK, Gregoriou GG, Savaki HE (2001) Functional imaging of the primate superior colliculus during saccades to visual targets. Nat Neurosci 4:1026-1031.

Moschovakis AK, Gregoriou GG, Ugolini G, Doldan M, Graf W, Guldin W, Hadjidimitrakis K, Savaki HE (2004) Oculomotor areas of the primate frontal lobes: a transneuronal transfer of rabies virus and $\left[{ }^{14} \mathrm{C}\right]-2-$ deoxyglucose functional imaging study. J Neurosci 24:57265740 .

Mushiake H, Fujii N, Tanji J (1999) Microstimulation of the lateral wall of the intraparietal sulcus compared with the frontal eye field during oculomotor tasks. J Neurophysiol 81:1443-1448.

Savaki HE, Raos VC, Dalezios Y (1997) Spatial cortical patterns of metabolic activity in monkeys performing a visually guided reaching task with one forelimb. Neuroscience 76:1007-1034.

Schall JD, Morel A, King DJ, Bullier J (1995) Topography of visual cortex connections with frontal eye field in macaque: convergence and segregation of processing streams. J Neurosci 15:4464-4487.

Schluppeck D, Glimcher P, Heeger DJ (2005) Topographic organization for delayed saccades in human posterior parietal cortex. J Neurophysiol 94:1372-1384.

Sereno MI, Pitzalis S, Martinez A (2001) Mapping of contralateral space in retinotopic coordinates by a parietal cortical area in humans. Science 294:1350-1354.

Silver MA, Ress D, Heeger DJ (2005) Topographic maps of visual spatial attention in human parietal cortex. J Neurophysiol 94:1358-1371.

Sokoloff L, Reivich M, Kennedy C, Des Rosiers MH, Patlak CS, Pettigrew KS, Sakurada O, Shinohara M (1977) The $\left[{ }^{14} \mathrm{C}\right]$-deoxyglucose method for the measurement of local cerebral glucose utilization: theory, procedure, and normal values in the conscious and anesthetized albino rat. J Neurochem 28:879-916.

Sommer MA, Wurtz RH (2000) Composition and topographic organization of signals sent from the frontal eye field to the superior colliculus. J Neurophysiol 83:1979-2001.

Swisher JD, Halko MA, Merabet LB, McMains SA, Somers DC (2007) Visual topography of human intraparietal sulcus. J Neurosci 27:5326-5337.

Thier P, Andersen RA (1998) Electrical microstimulation distinguishes distinct saccade-related areas in the posterior parietal cortex. J Neurophysiol 80:1713-1735.

Van Gisbergen JA, Robinson DA, Gielen S (1981) A quantitative analysis of generation of saccadic eye movements by burst neurons. J Neurophysiol 45:417-442.

Wurtz RH, Goldberg ME (1972) Activity of superior colliculus in behaving monkey. III. Cells discharging before eye movements. J Neurophysiol 35:575-586. 